\title{
A model for quantifying genetic recombination in chromosome polymorphisms due to supernumerary heterochromatic segments
}

\author{
J. de la Torre, \\ E. Torroja*, \\ J. Gosálvez and \\ C. López-Fernández
}

\author{
Dpto. de Genética C-XV, Facultad de Ciencias, \\ Universidad Autónoma de Madrid, 28049 Madrid, \\ Spain. \\ * Centro de Investigaciones Biológicas, C.S.I.C., \\ Velázquez 144, 28006 Madrid, Spain.
}

Heterochromatic supernumerary segments can modify chiasma distribution in the affected bivalents. A model is developed for quantifying the genetic recombination in populations polymorphic for such segments. Ihis paper describes this model for a monochiasmate bivalent, and its application in different populations of the grasshopper Chorthippus parallelus. The observations suggest that these polymorphic systems have evolutionary implications.

\section{INTRODUCTION}

During the past years many cytogeneticists and molecular biologists have focused their attention on the study of heterochromatin, a chromosomal material first described by Heitz (1928). Although we still ignore many aspects related to its composition and function, many authors have reported a correlation between its presence in a genome and variations in the frequency and distribution of chiasmata. Thus, John (1981) claimed that "there are grounds for arguing that one of the functions of the heterochromatin variations to be found in natural populations is the regulation of recombination and, through this, the regulation of the variation present within and between populations".

The pattern of recombination is considered to play an important role in the maintenance of coadapted gene complexes within chromosomes (Darlington, 1939; Wallace, 1953). Therefore the heterochromatin, by modifying recombination, can potentially produce important effects on populations. In this regard, many animal species, particularly the grasshoppers, show large heterochromatic segments added to one or several chromosomes of their complements. In many cases this extra material introduces chromosome polymorphisms within a population, giving rise to different karyomorphs which usually differ greatly in their patterns of chiasma distribution (John, 1981; García-Lafuente et al., 1983; John and King,
1985; Navas-Castillo et al., 1985; Rufas et al., 1986). However, in no case has a model been described or established in order to quantify the effect of polymorphic heterochromatic supernumerary segments on the production of recombinants, in terms of the frequency of the segments in a given population.

\section{THE MODEL}

The aim of this paper is to generalise a model (de la Torre et al., 1986) to quantify the rate of recombination within determined zones of a chromosome affected by heterochromatic segments for a given population, taking into account the pattern of chiasma distribution and the frequency of each karyomorph in that population. Although the model can be extended to the analysis of more complex polymorphic systems, we will describe here the simplest system, i.e., the consequences of a distal supernumerary segment in a monochiasmate bivalent, where chromatid and chiasma interference can be disregarded (Sybenga, 1975, p. 20). In these bivalents the single chiasma can be localised in different sections of the chromosome (proximal, interstitial and distal) (fig. 1). When one supernumerary segment is segregating in the population three different karyomorphs (i) are expected (fig. 1). These karyomorphs are named as follows: 


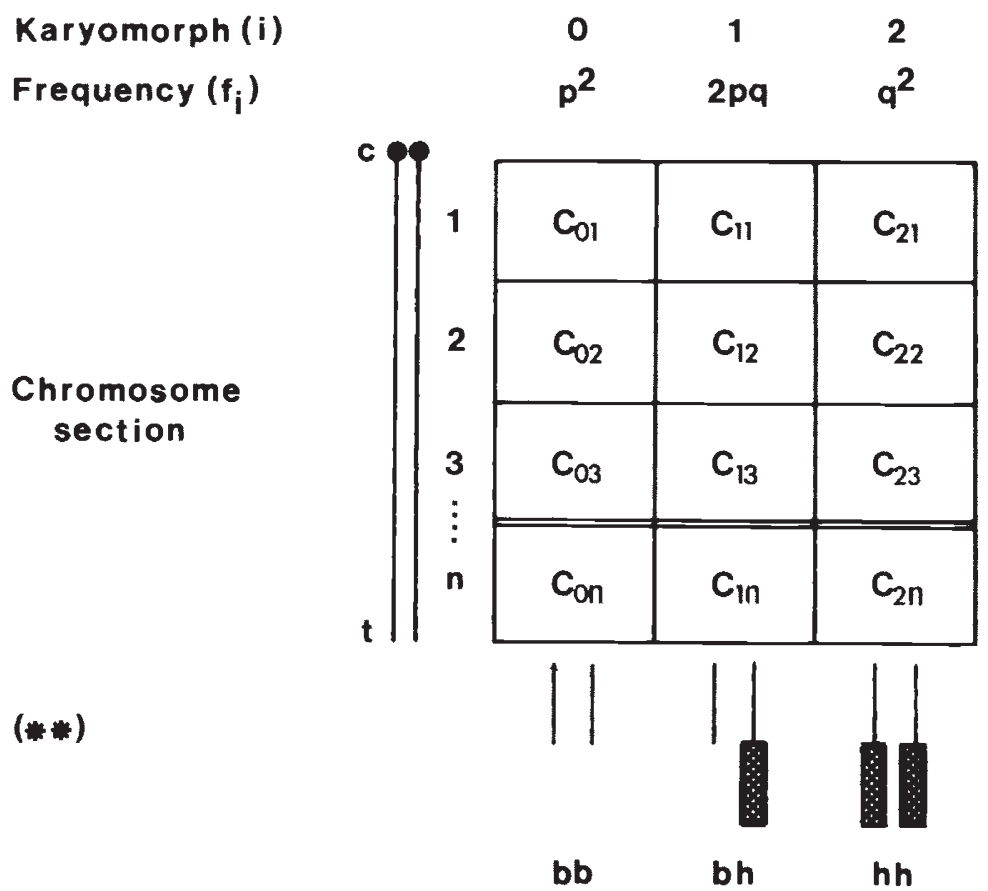

Figure 1 Representation of the different chromosome sections $(1,2 \ldots n)$ considered in each karyomorph $(0,1,2)$. The $C_{i j}$ value (see text) for each case is also represented. $(* *)$ corresponds to a diagrammatic representation of the heterochromatic content per karyomorph $(b b, b h$ and $h h)$.

(a) karyomorph type $i=0$ : basic homozygote; without heterochromatic segments $(b b)$.

(b) karyomorph type $i=1$ : heterozygote; with one heterochromatic segment $(b h)$.

(c) karyomorph type $i=2$; structural homozygote; with two heterochromatic segments $(h h)$.

The segment segregating in the population can be considered as a mendelian unit; and, according to the Hardy-Weinberg law, the expected frequencies of the three karyomorphs in equilibrium $(f i)$ will be as follows:

$$
\begin{aligned}
& f_{0}=p^{2}: \text { karyomorph type } i=0(b b) \\
& f_{1}=2 p q: \text { karyomorph type } i=1(b h) \\
& f_{2}=q^{2}: \text { karyomorph type } i=2(h h) .
\end{aligned}
$$

By means of a quantitative analysis of the pattern of chiasma distribution at diplotene in the affected bivalent one obtains the value $C_{i j}$ which expresses the frequencies of the single chiasma in different (and predetermined) chromosome sections $(j)$ for the three karyomorphs $(i)$. For example, $C_{01}$ corresponds to the frequency of chiasmata located in section 1 of bivalents from basic homozygotes $(i=0)$ (see fig. 1).
Taking into account these values the expected chiasma distribution in a population can be calculated as a function of the frequency of the heterochromatic segment $(q)$. This will be an addition of the contributions of each karyomorph for each kind of chiasma. In the case of section $j$ it will have the following expression:

$$
Q_{j}=\sum_{i=0}^{2} f_{i} C_{i j}
$$

Thus, for instance, the frequency of chiasma formation in section 1 in the population has the value,

$$
Q_{1}=f_{0} C_{01}+f_{1} C_{11}+f_{2} C_{21}
$$

where each term represents the contribution of each karyomorph to the total frequency of chiasmata in that section. The term $f_{1} C_{11}$ reflects the exchange in this section in a heteromorphic bivalent.

On the other hand, since only two of the four chromatids of a bivalent are involved in the formation of a single chiasma, the frequency of recombinant gametes resulting from a chiasma in section $j$ is given by the expression,

$$
R_{j}=1 / 2 Q_{j}=1 / 2 \sum_{i=0}^{2} f_{i} C_{i j} .
$$


The term $1 / 2 f_{1} C_{1 j}=p q C_{1 j}$ expresses the frequency of recombinant gametes in heteromorphic bivalents.

Whenever the $C_{i j}$ value is the same among different populations, i.e., the supernumerary segment provokes an indentical effect irrespective of the population, the expression (3) can be plotted against the frequency of the heterochromatic segment in the population ( $o \leqq q \leqq 1$ ) (see fig. 2 in our example). Thus, this bidimensional representation permits us to assess the differences in the contribution of distinct recombinant gametes of several populations according to their frequencies of a given heterochromatic segment $(q)$.

\section{THE EXAMPLE}

To illustrate the model, we have used a grasshopper widespread throughout Europe (Chorthippus parallelus) for which information on the distribution of heterochromatic segments in different populations, and on the chiasma distribution within the affected bivalents, is available. This species presents a polymorphic system of supernumerary segments affecting the $M_{7}$ and the $S_{8}$ chromosomes. In this paper we have considered the data on the presence of supernumerary segments in the $S_{8}$ bivalent of this species, as reported by Hewitt and John (1968) and Westerman (1969), for the populations of Boulogne-Sur-Mer (Bm), Col de Pourtalet $(\mathrm{Cp})$ and Foret d'Ecouves (Fe) in France and for the populations of Ashurst (As), Ystradgynlais (Yg2), Port Madoc (Pm), Holway Farm (Hf) and Staple Hill (Sh) in England. The genotypic frequencies $(b b, b h, h h)$ observed for each case are summarised in table 1 .

Table 1 Genotypic frequencies observed for the populations considered in the example. Data from Hewitt \& John (1968) and Westerman (1969)

\begin{tabular}{llll}
\hline & \multicolumn{3}{c}{ Genotypic frequencies } \\
& $p^{2}(b b)$ & $2 p q(b h)$ & $q^{2}(h h)$ \\
\hline French populations & & & \\
Boulogne-Sur-Mer(Bm) & 0.83 & 0.17 & 0.00 \\
Col de Pourtalet (Cp) & 0.45 & 0.50 & 0.05 \\
Foret d'Ecouves (Fe) & 0.68 & 0.29 & 0.03 \\
British populations & & & \\
Ashurst (As) & 0.55 & 0.33 & 0.12 \\
Ystradgynlais (Yg2) & 0.32 & 0.42 & 0.26 \\
Port Madoc (Pm) & 0.40 & 0.33 & 0.27 \\
Holway Farm (Hf) & 0.68 & 0.28 & 0.04 \\
Staple Hill (Sh) & 0.95 & 0.05 & 0.00 \\
\hline
\end{tabular}
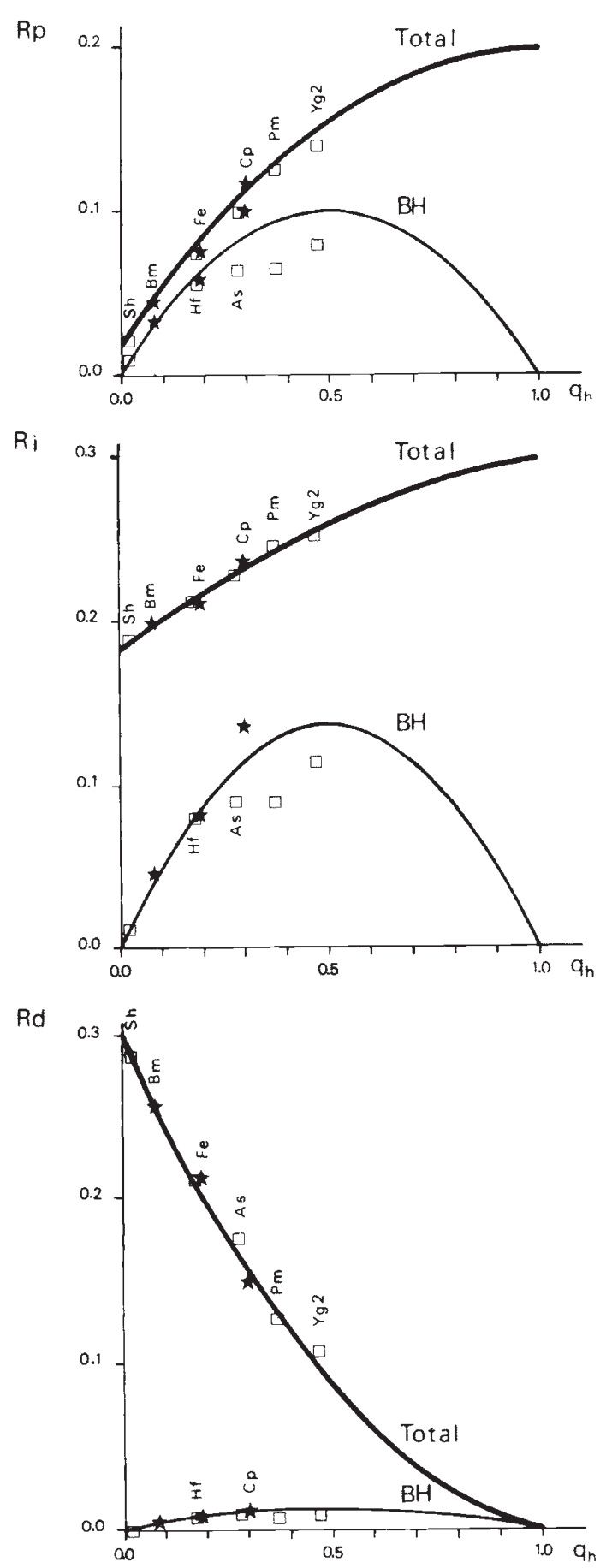

Figure 2 Representation of the $R_{j}$ functions depending on the $q$ frequency in the population for the $S_{8}$ chromosome of Chorthippus parallelus. $R_{p}$ : proximal chiasma, $R_{i}$ : interstitial chiasma, $R_{d}$ : distal chiasma. Total and $B H$ in each case represents the frequency of gametes produced by recombination in each section considering the three karyomorphs in the population (Total) or only the heterozygotes $(B H)$. Stars and squares represent French and British populations respectively. 
The $S_{8}$ bivalent can be divided in three sections (proximal, interstitial and distal) where the single chiasma is formed. The karyomorphs $b b, b h$ and $h h$ present significant differences in their chiasma distribution. Basic homozygotes $(b b)$ show a high frequency of distal chiasmata. On the contrary, in both heterozygotes $(b h)$ and structural homozygotes $(h h)$ the incidence of interstitial and proximal chiasmata is notably increased (NavasCastillo et al., 1985). When comparing these results with our observations from other populations (Gosálvez, unpublished) we have not obtained any significant differences. This means that in any population this supernumerary segment exerts an identical effect on the chiasma distribution. Therefore, we can apply the available results on chiasma distribution from some populations to the observations on the frequency of the segment in other populations.

The function $R_{j}$ for the three sections considered in the $S_{8}$ chromosome (proximal, interstitial, distal) (fig. 2) has been obtained from the data of the chiasma distribution in the three classes (bb, bh, hh) of $S_{8}$ bivalents of Chorthippus parallelus. The contribution of the three karyomorphs (Total in fig. 2), as well as the curve which reflects the exchange in heteromorphic bivalents (BH in fig. 2), have been plotted for all the sections ( $\boldsymbol{R}_{p}$-proximal, $\boldsymbol{R}_{i}$-interstitial, $\boldsymbol{R}_{d}$-distal). In both cases the curves are based on the expected genotypic frequencies for whichever gene frequency ( $o \leqq q \leqq 1$ ). Moreover, we can also represent the analysed populations (see table 1) according to their observed genotypic frequencies. The populations do not necessarily fit in the theoretical curve. These deviations arise from the differences in the genotypic frequencies among the populations since the chiasma distribution for each karyomorph ( $C_{i j}$ values) is identical for each population. According to this model, it is obvious that in a population affected by polymorphic supernumerary segments where the distinct karyomorphs differ with respect to the pattern of chiasma distribution, the pattern of recombinant products in the population will depend on the frequency of each kind of bivalent. Therefore, two populations with different frequencies of karyomorphs will produce unequal frequencies of the different recombinant products and these differences can be quantified.

\section{FURTHER CONSIDERATIONS}

The modification of the pattern of chiasma distribution according to the presence or absence of supernumerary heterochromatin induces some changes in the proportion of the different variants among the gametes generated by the population. Although our analysis does not permit the identification of the chromosome region influenced by the heterochromatin, it can be postulated that this system works in the way depicted in fig. 3. That is, in heterozygotes chiasma formation will be nearly suppressed in the $\alpha$-region while the frequency of chiasmata is increased in the $\beta$ and $\gamma$ regions. This effect leads to the permanence of a certain block of genes located in the $\alpha$-region and adjacent to the segment. On the contrary recombination will subject $\beta$ and $\gamma$ regions to variations.

Two situations have been described with respect to structural homozygotes:

(a) Structural homozygotes present a chiasma distribution similar to that shown by

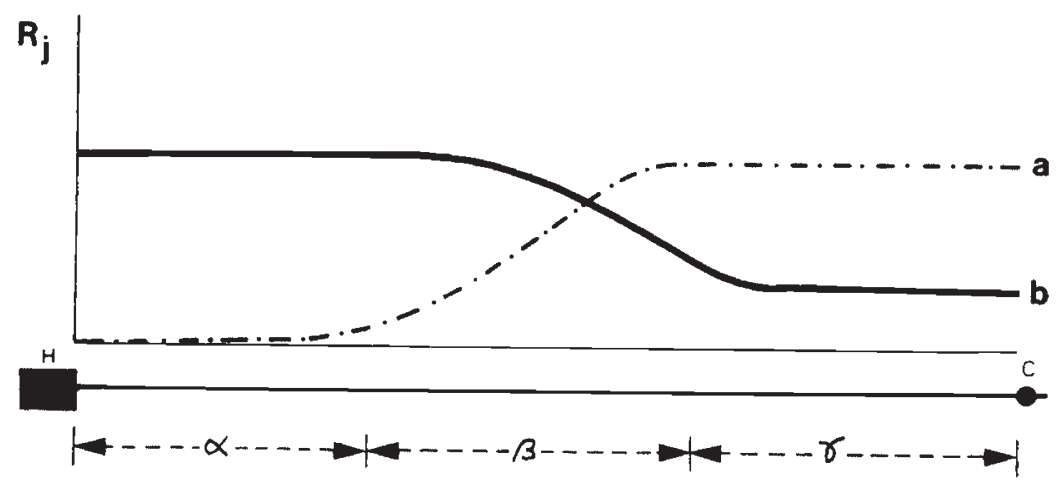

Figure 3. Representation of the chiasma readjusting due to the presence of a heterochromatic segment $(H)$. "a" and " $b$ " correspond to the behaviour of a heterozygous or basic homozygous bivalents respectively. Structural homozygotes may behave as "a" or " $b$ " depending on the analyzed system (see text). $\alpha, \beta$ and $\gamma$ represent hypothetical regions in a bivalent where a chiasma can be formed. $C$ : centromere. 
heterozygotes. That is suppression of recombination in the $\alpha$-region (John, 1981; and this exampie).

(b) Structural homozygotes present a chiasma distribution similar to that shown by basic homozygotes. That is a high frequency of chiasmata in the $\alpha$-region (de la Torre et al., 1986).

In the first case, the block of genes near the heterochromatin remain linked both in heterozygotes and structural homozygotes. Consequently, a hypothetical advantage of the block of genes adjacent to the heterochromatin would be preserved but its evolution would be very slow.

In the second case, there is free recombination in the $\alpha$-region which would favour the establishment of flexible polymorphisms with local adaptation. If the gene complexes included in the $\alpha$ region present certain adaptive advantages, the frequency of chromosomes carrying supernumerary segments would increase to form a consistent local polymorphism. When the frequency of this segment reaches a certain value in the population, in the structural homozygotes the genetic material included in the $\alpha$-region could suffer a process of remodelling under the pressure of natural selection, similar to that occurring in the case of inversion polymorphisms (Powell et al., 1973).

In both cases polymorphisms are generated but their characteristics are clearly distinct and their possibilities of stabilisation and evolution are predicted to be different.

These considerations are, of course, consistent if it is accepted that these polymorphisms have some importance for the evolution of the genetic system of a given species. Unfortunately this fact awaits validation. The alternative, which suggests that supernumerary segments have no evolutionary potential, i.e., they are transient and neutral in terms of evolution, has not been experimentally rejected.
Acknowledgements The authors sincerely thank Dr J. Sybenga and Dr C. García de la Vega for valuable discussions. The investigation was supported by C.A.I.C.Y.T. $(2165 / 83)$.

\section{REFERENCES}

DARLINGTON, C. D. 1939. The evolution of genetic systems, Cambridge Univ. Press.

GARCIA-LAFUENTE, R., LOPEZ-FERNANDEZ, C. AND GOSALVEZ, J. 1983. Extra heterochromatin in natural populations of Gomphocerus sibiricus (Orthoptera: Acrididae). 2. Chiasma distribution in the M7 bivalent. Cytobios, 37, 149-155.

HEITZ, E. 1928. Das heterochromatin der Moose. Jb. Wiss. Bot, $69,762-818$.

HEWITT, G. M. AND JOHN, B. 1968. Parallel polymorphism for supernumerary segments in Chorthippus parallelus (Zetterstedt). I. British populations. Chromosoma, 25, 319-342.

JOHN, B. 1981. Heterochromatin variation in natural populations, Chromosomes today, 7, George Allen \& Unwin, pp. 128-137.

JOHN, B. AND KING, M. 1985. The inter-relationship between heterochromatin distribution and chiasma distribution. Genetica, 66, 183-194.

NAVAS-CASTILlo, J, CABRERo, J. AND CAMACHO, J. P. M. 1985. Chiasma redistribution in bivalents carrying supernumerary chromosome segments in grasshoppers. Heredity, $55,245-248$.

POWEll, J. R., LEVENE, H. AND DOBZHANSKY, TH. 1973. Chromosomal polymorphism in Drosophila pseudoobscura used for diagnosis of geographic origin. Evolution, 26, 553-559.

RUFAS, J. S., SUJA, J. A. AND GARCIA DE LA VEGA, C. 1986. Generation by a polymorphic supernumerary segment of recombination in a normally achiasmate proximal region in Acrotylus insubricus (Scopoli) (Orthoptera: Acrididae). Canad. J. Genet. Cytol., 28, 433-438.

SYBENGA, J. 1975. Meiotic configurations, Springer-Verlag, Berlin, Heidelberg, New York, p. 20.

TORRE, J. DE LA, LOPEZ-FERNANDEZ, C., NICHOLS, R. AND GOSALVEZ, J. 1986. Heterochromatin readjusting chiasma distribution in two species of the genus Arcyptera: The effect among individuals and populations. Heredity, 56, $177-184$.

Wallace, B. 1953. On coadaptation in Drosophila. Amer. Nat., $87,343-358$

WESTERMAN, M. 1969. Parallel polymorphism for supernumerary segments in Chorthippus parallelus. II. French populations. Chromosoma, 26, 7-21. 